Platinum-based chemotherapy was concurrently administered for $44(62 \%)$ patients. 3/5-year overall survival (OS) rate and progression-free survival (PFS) rate were $64 / 50 \%$ and $40 / 37 \%$, respectively. Membranous expression of PD-L1 was positive in $8.5 \%$ of patients, and not associated with prognosis.

The presence of tumor-infiltrating $\mathrm{T}$ cell positive for CD8 (CD8+TILs) in the tumor nest was positive in $83 \%$ of patients, and associated with favorable OS $(\mathrm{p}=0.037)$ but not with PFS $(p=0.52)$. A Cox regression analysis showed that the presence of CD8+TILs was an independent predictor of favorable OS (hazard ratio $=0.19 ; 95 \%$ confidence interval $=0.065-0.57$ ) as well as lower International Federation of Gynecology and Obstetrics stage (2008, I-II vs. III-IVA), maximum tumor diameter smaller than $40 \mathrm{~mm}$, and use of concurrent chemotherapy.

Conclusion: We reported clinical outcomes of CA after definitive $\mathrm{RT}$ and expression of immune-related molecules using biopsy sample. Our findings suggested that CD8+TILs in the tumor nest was a potential predictor of favorable prognosis, although prognostic impact of membranous PD-L1 expression was unclear.

Oral (OC7)

Preinvasive Disease of Cervix, Vulva, and Vagina

https://doi.org/10.3802/jgo.2021.32.S1.0C7

\section{Prognostic outcomes and risk factors for recurrence after laser vaporization for cervical intraepithelial neoplasia: a single- center retrospective study}

Keisuke Kodama, ${ }^{1}$ Hideaki Yahata, ${ }^{1, *}$ Kaoru Okugawa, ${ }^{1}$ Hiroshi Yagi, ' Masafumi Yasunaga,' Tatsuhiro Ohgami, Ichiro Onoyama, ${ }^{1}$ Kazuo Asanoma, ${ }^{1}$ Mototsugu Shimokawa, ${ }^{2}$ Kiyoko Kato'

'Kyushu University, Fukuoka, Japan (hyahata@med.kyushu-u.ac.jp) ${ }^{2}$ Yamaguchi University, Yamaguchi, Japan

Objective: Cervical intraepithelial neoplasia (CIN) is a precancerous lesion that may progress to invasive cervical cancer without intervention. We aim to examine the prognostic outcomes and risk factors for recurrence after laser vaporization for CIN 3, CIN 2 with high-risk human papillomavirus (HPV) infection, and CIN 1 persisting for more than 2 years.

Methods: Between 2008 and 2016, a total of 1,070 patients underwent cervical laser vaporization using a carbon dioxide laser. We performed a retrospective review of their medical records to assess their clinical characteristics, pathologic factors, and prognostic outcomes.

Results: The mean patient age was 34 years (range, 18-64 years). The preoperative diagnosis was CIN 1 in 27 patients, CIN 2 in 485 patients, and CIN 3 in 558 patients. Over a median follow-up period of 15 months, the 2-year recurrence rate was $18.9 \%$, and the 5 -year recurrence rate was $46.5 \%$. The 2 -year retreatment rate was $12.6 \%$, and the 5 -year retreatment rate was 30.5\%. We diagnosed 9 patients with invasive cancer after treatment; all patients underwent combined multidisciplinary treatment, and there were no deaths during follow-up. The recurrence-free interval was correlated with patient age (hazard ratio $[\mathrm{HR}]=1.028$; 95\% confidence interval $[\mathrm{CI}]=1.005-1.051$; $\mathrm{p}=0.0167)$, body mass index $(\mathrm{HR}=1.052 ; 95 \% \mathrm{CI}=1.008-1.098$; $\mathrm{p}=0.0191)$, and glandular involvement $(\mathrm{HR}=1.962 ; 95 \%$ $\mathrm{CI}=1.353-2.846$; $\mathrm{p}=0.0004$ ).

Conclusion: Cervical laser vaporization is effective and useful for patients with CIN who wish to preserve fertility. However, patients with glandular involvement, older age, and higher body weight require close follow-up for recurrence.

\section{Uterine corpus cancer}

Oral (OM1)

Endometrial Hyperplasia, Endometrial Intra-epithelial Neoplasia, and Endometrial Cancer

https://doi.org/10.3802/jgo.2021.32.S1.0M1

\section{The real-world experience of pembrolizumab and lenvatinib in recurrent endometrial cancer: a multicenter study in South Korea}

\footnotetext{
Junhwan Kim, ${ }^{1}$ Joseph J. Noh, ${ }^{2}$ Tae Kyoung Lee, ${ }^{3}$ Se Ik Kim, Jung-Yun Lee, ${ }^{3}$ Jeong-Won Lee, ${ }^{2}$ Jae-Weon Kim ${ }^{1, *}$

'Department of Obstetrics and Gynecology, Seoul National University College of Medicine, Seoul, Korea (kjwksh@snu.ac.kr)

${ }^{2}$ Samsung Medical Center, Sungkyunkwan University School of Medicine, Seoul, Korea

${ }^{3}$ Department of Obstetrics and Gynecology, Women's Cancer Center, Institute of Women's Life Medical Science, Yonsei University College of Medicine, Seoul, Korea
}

Objective: To investigate real-world experience of pembrolizumab and lenvatinib (PEMBRO+LEN) in patients with recurrent endometrial cancer in South Korea.

Methods: This retrospective cohort study included patients with recurrent endometrial cancer who received PEMBRO+LEN from January 2020 to May 2021 in three tertiary hospitals in South Korea. We summarized patient characteristics and evaluated response rates, survival outcomes, and treatment-related adverse events (AEs).

Results: In total, 48 patients were included in the study. The median age was 62.5 (42-78) years. The most common histologic subtype was endometrioid $(43.8 \%)$ followed by serous (25.0\%). Most patients were MMR proficient (91.7\%). Programmed death-ligand 1 and P53 status were positive 
in $29.2 \%$ and $18.8 \%$, respectively. Best objective response rate was $20.8 \%$ and disease control rate was $66.6 \%$. Median progression-free survival (PFS) was 5.3 (95\% confidence interval=3.9-6.6), respectively. The median number of cycles for PEMBRO+LEN was 4.5. More than half of patients (56.2\%) received LEN dose reduction was once or more but it did not affect survival outcomes. Common AEs of LEN was fatigue $(18.8 \%)$, hypertension $(16.7 \%)$ and hypothyroidism (14.6\%). Treatment-free interval (TFI) was the only statistically significant factor associated with PFS $(\mathrm{p}=0.017)$. In a subgroup analysis, TFI 5 months showed statistically higher in best overall response $(\mathrm{p}=0.042)$ and in the number of the cycle of AEs first encountered during PEMBRO+LEN treatment ( $\mathrm{p}=0.017)$.

Conclusion: In Korean patients with recurrent endometrial cancer, PEMBRO+LEN treatment showed acceptable response rates with tolerable AEs when treatment was supported with optimal LEN dose reduction.

Oral (OM2)

Endometrial Hyperplasia, Endometrial Intra-epithelial Neoplasia, and Endometrial Cancer

https://doi.org/10.3802/jgo.2021.32.S1.0M2

\section{Menopausal status combined with serum CA125 level significantly predicted concurrent endometrial cancer in women diagnosed with atypical endometrial hyperplasia before surgery}

\section{Yaochen Lou, Jiongbo Liao, Jun Guan*}

Obstetrics and Gynecology Hospital of Fudan University, Shanghai, China (junguan2018@163.com)

Objective: About $10 \%-60 \%$ of patients with atypical endometrial hyperplasia diagnosed before surgery (preoperative-AEH) are found to have concurrent endometrial cancer (EC) at definitive hysterectomy, leading to incomplete primary surgery and delayed adjuvant treatment. This study aims to investigate the potential risk factors of concurrent EC in preoperative-AEH patients in a clinical setting with gynecological pathology review.

Methods: All patients diagnosed with $\mathrm{AEH}$ by endometrial biopsy or curettage and then underwent definitive hysterectomy from January 2016 to December 2019 were analyzed. All diagnoses were reviewed by gynecological pathologists. Results: Totally 624 preoperative-AEH patients were included, $30.4 \%$ of whom had concurrent EC. In multivariate analysis, postmenopausal status and cancer antigen 125 (CA125) $\geq 35$ $\mathrm{U} / \mathrm{mL}$ significantly correlated with concurrent EC (odds ratio
$[\mathrm{OR}]=3.33,95 \%$ confidence interval $[\mathrm{CI}]=1.76-6.30 ; \mathrm{OR}=2.12$, $95 \% \mathrm{CI}=1.13-3.98)$. Similar findings were showed in predicting intermediate-high-risk EC. The combined predicting value of postmenopausal status and CA125 $\geq 35 \mathrm{U} / \mathrm{mL}$ were remarkably increased for concurrent $\mathrm{EC}(\mathrm{OR}=14.10,95 \% \mathrm{CI}=1.59-125.22)$ and intermediate-high-risk EC $(\mathrm{OR}=30.2$, 95\% $\mathrm{CI}=2.44-$ 374.40). Notably, concurrent intermediate-high-risk EC was more commonly seen in preoperative-AEH women with postmenopausal time $\geq 5$ years $(\mathrm{OR}=7.83,95 \% \mathrm{CI}=2.44-25.18$, $\mathrm{p}=0.001$ ), with no difference in this risk among subgroups of premenopausal status, postmenopausal time $<2$ years or between 2-5 years.

Conclusion: Preoperative-AEH patients with postmenopausal status and elevated level of CA125 may have high risk of concurrent EC. Adequate pre-surgical evaluation and sentinel lymph node sampling should be suggested for such patients.

Oral (OM3)

Endometrial Hyperplasia, Endometrial Intra-epithelial Neoplasia, and Endometrial Cancer

https://doi.org/10.3802/jgo.2021.32.S1.0M3

\section{Clinical implications of neoadjuvant chemotherapy in advanced endometrial cancer: a multi-center retrospective cohort study}

\author{
Hyunji Lim, ${ }^{1}$ Se Ik Kim, ${ }^{1}$ Tae Hun Kim, ${ }^{2}$ Dong Hoon Suh, ${ }^{3}$ \\ Myong Cheol Lim, ${ }^{4}$ Jae-Weon Kim ${ }^{1, *}$ \\ 'Seoul National University College of Medicine, Seoul, Korea \\ (kjwksh@snu.ac.kr) \\ ${ }^{2}$ Seoul Metropolitan Government-Seoul National University Boramae \\ Medical Center, Seoul, Korea \\ ${ }^{3}$ Seoul National University Bundang Hospital, Seongnam, Korea \\ ${ }^{4}$ National Cancer Center, Goyang, Korea
}

Objective: In a portion of patients with advanced endometrial cancer, upfront complete cytoreduction is not possible. Hence, we aimed to assess the feasibility and effectiveness of neoadjuvant chemotherapy followed by interval debulking surgery (NAC-IDS) in unresectable, metastatic endometrial cancer. We also investigated factors which related to the favorable conditions for NAC-IDS in endometrial cancer. Methods: From the four institutions in Korea, we identified stage IIIC-IVB endometrial cancer patients who received NACIDS between 2008 and 2020. We collected patients' baseline characteristics, NAC regimens, response to NAC, and surgical details. Both progression-free survival (PFS) and overall survival (OS) were calculated, and factors affecting survival outcomes were investigated. 